\title{
Eficiência de malhas amostrais utilizadas na caracterização da variabilidade espacial de fósforo e potássio
}

\author{
Efficiency of sampling grids used in the characterization of phosphorus and potassium

\begin{abstract}
Maurício Roberto Cherubin ${ }^{\mathrm{I}^{*}}$ Antônio Luis Santi ${ }^{\mathrm{II}}$ Mateus Tonini Eitelwein ${ }^{\mathrm{I}}$ Diego Ricardo MenegolII Clóvis Orlando Da Ros ${ }^{\mathrm{II}}$ Osmar Henrique de Castro Pias ${ }^{\mathrm{II}}$ Juliano Berghetti ${ }^{\mathrm{II}}$
\end{abstract}

RESUMO

O objetivo do trabalho foi estudar a eficiência das malhas amostrais, utilizadas nas áreas manejadas com agricultura de precisão, para a caracterização da variabilidade espacial dos teores de fósforo $(P)$ e potássio (K). O estudo foi conduzido em 30 áreas agrícolas, localizadas no Rio Grande do Sul, cujos solos foram classificados como Latossolos Vermelhos. As dimensões das malhas amostrais estudadas foram: 100×100m (10 áreas), $142 \times 142 m$ (10 áreas) e 173×173m (10 áreas). Foram analisados os teores de $P$ e K na profundidade de 0,00-0,10m. Os dados foram submetidos à análise estatística descritiva e a análise geoestatística. De modo geral, as áreas estudadas apresentaram teores médios de $P$ e K adequados ao desenvolvimento das culturas. Entretanto, observaram-se locais com baixos teores de $P$, justificando fertilizações em sítio-específico. Com base em parâmetros geoestatísticos, as dimensões de malhas amostrais utilizadas são eficientes em captar as diferentes escalas da variabilidade espacial de $P$ e $K$, porém, recomenda-se o uso de malhas amostrais menores, que considerem a variabilidade espacial a curtas distâncias.

Palavras-chave: agricultura de precisão, amostragem de solo, fertilidade do solo, geoestatística, Latossolos.

\section{ABSTRACT}

The objective of the research was to evaluate the efficiency of the sampling grids, used in areas managed with precision agriculture, for the characterization of spatial variability levels of phosphorus (P) and potassium (K). The study was conducted in 30 agricultural areas, located in Rio Grande do Sul state, presenting soils classified as Oxisols. The sizes of the

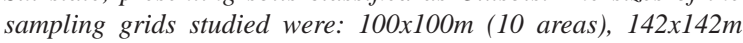
(10 areas) and $173 \times 173 m$ (10 areas). Were analyzed the levels of $P$ and $K$, in depth of sampling from $0.00-0.10 m$. The data were subjected to descriptive statistical analysis and geostatistical analysis. In, general, the areas showed average levels of $P$ and $K$ suitable for crops development. However, were observed subareas with low levels of $P$, justifying fertilization on site-specific. Based on geostatistical parameters, sizes of the sampling grids used are efficient to capture the different scales of spatial variability of $P$ and $K$, however, it is indicated smaller sampling grids to consider the spatial variability over short distances.

Key words: precision agriculture, soil sampling, soil fertility, geostatistical, Oxisols.

\section{INTRODUÇÃO}

A adoção da agricultura de precisão (AP), na região Sul do Brasil, tem se intensificado nos últimos anos. Estima-se que, somente no Rio Grande do Sul, mais de dois milhões de hectares (ha) utilizem alguma ferramenta de AP (SANTI et al., 2009), especialmente aquelas voltadas ao manejo da fertilidade do solo.

A eficiência do manejo da fertilidade do solo, realizado nas áreas de AP, é diretamente condicionada pela qualidade da amostragem de solo, tradicionalmente realizada de modo sistemático por meio de malhas regulares (KERRY et al., 2010; WEBSTER \& LARK, 2012), a qual deve possibilitar captar as diferentes escalas de variabilidade espacial dos atributos do solo (BOTTEGA et al., 2013). Para tanto, a definição da dimensão da malha amostral, necessariamente, deve levar em consideração

IEscola Superior de Agricultura "Luiz de Queiroz" (ESALQ), Universidade de São Paulo (USP), Avenida Pádua Dias, 11, 13418-900 Piracicaba, SP, Brasil. E-mail: cherubin@usp.br.*Autor para correspondência.

IIDepartamento de Ciências Agronômicas e Ambientais, Centro de Educação Superior Norte do Rio Grande do Sul, Universidade Federal de Santa Maria (UFSM), campus de Frederico Westphalen, Frederico Westphalen, RS, Brasil. 
princípios geoestatísticos, como a dependência espacial dos atributos de solo estudados (WEBSTER \& OLIVER, 2007; KERRY et al., 2010). Entretanto, o que se tem observado comercialmente nas áreas manejadas com AP no sul do Brasil é a utilização de malhas regulares, com amostras espaçadas por $100 \mathrm{~m}$ (uma amostra a cada um ha) a $175 \mathrm{~m}$ (uma amostra a cada 3ha), definidas basicamente por razões de ordem econômica e prática.

Diversos estudos têm demonstrado que a dependência espacial dos atributos é variável conforme o tipo de solo (MONTANARI et al., 2008), relevo (SOUZA et al., 2006; MONTANARI et al., 2008), atributo estudado (CHERUBIN et al., 2011; NANNI et al., 2011), distância entre amostras (KERRY et al., 2010; NANNI et al., 2011) e uso e manejo do solo (BOTTEGA et al., 2013). Portanto, as diferentes escalas de variação dos atributos do solo induzem grande dificuldade no desenvolvimento de um plano de amostragem, que utilize uma malha amostral com espaçamento único, quando vários atributos do solo estão envolvidos (MONTANARI et al., 2012).

Nesse contexto, objetivou-se estudar a eficiência das malhas amostrais, utilizadas em áreas de Latossolos Vermelhos manejadas com AP, na caracterização da variabilidade espacial do fósforo (P) e potássio $(\mathrm{K})$.

\section{MATERIAL E MÉTODOS}

O estudo foi conduzido em 30 áreas agrícolas, localizadas na região Norte do Rio Grande do Sul, Brasil, abrangendo $2.467,82$ ha. A região estudada está localizada na província geomorfológica do Planalto, com relevo suave ondulado. Os solos predominantes foram classificados como Latossolos Vermelhos de classe textural argilosa, profundos, com alta acidez e baixa fertilidade natural (STRECK et al., 2008).

O manejo adotado nas áreas é o sistema plantio direto, com cultivos rotacionados de soja e milho nas safras de verão e aveia branca (grãos), aveia preta e nabo forrageiro (cobertura do solo) nas safras de inverno.

As áreas foram amostradas a partir das dimensões de malhas amostrais que comumente são utilizadas em nível de campo no sul do Brasil, sendo

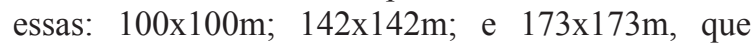
correspondem a uma amostra a cada um, dois e três ha, respectivamente. Assim, cada malha amostral foi utilizada em 10 áreas, totalizando 1.461 amostras de solo.
As amostras foram compostas por 10 subamostras, coletadas em um raio de $10 \mathrm{~m}$ dos pontos georreferenciados, utilizando um trado de rosca acoplado a um quadriciclo, na profundidade de 0,00-0,10m. Esse procedimento de coleta de solo segue o que está sendo realizado atualmente nas áreas de AP da região estudada. Posteriormente, os teores de $\mathrm{P}\left(\mathrm{mg} \mathrm{dm}^{-3}\right)$ e $\mathrm{K}\left(\mathrm{mg} \mathrm{dm}^{-3}\right)$ foram extraídos por Mehlich-1, seguindo metodologias recomendadas pela CQFS-RS/SC (2004). Quanto aos parâmetros interpretativos de $\mathrm{P}$ e $\mathrm{K}$, as áreas apresentaram teores médios de argila classificados na classe 2 (40-60\%) e capacidade de troca de cátions potencial $\left(\mathrm{CTC}_{\mathrm{pH} 7,0}\right)$ na classe média $\left(5,1-15,0 \mathrm{cmol}_{\mathrm{c}} \mathrm{dm}^{-3}\right)$.

Os dados foram submetidos à análise estatística descritiva no programa Statistical Analysis System - SAS 8.0 (SAS Inc, Cary, EUA). A análise da variabilidade espacial dos dados de $\mathrm{P}$ e $\mathrm{K}$ foi realizada por meio de semivariogramas, cujos ajustes foram realizados por modelos matemáticos teóricos, utilizando o programa computacional GS+ (Gamma Design Software, LLC, Plainwell, EUA). Os modelos dos semivariogramas foram ajustados com base na menor soma de quadrados do resíduo (SQR) e no melhor coeficiente de determinação $\left(\mathrm{r}^{2}\right)$, e avaliados pela técnica de validação cruzada. Do ajuste de um modelo matemático aos dados, foram definidos os parâmetros do semivariograma: efeito pepita $\left(\mathrm{C}_{0}\right)$, contribuição $\left(\mathrm{C}_{1}\right)$, patamar $\left(\mathrm{C}_{0}+\mathrm{C}_{1}\right)$ e alcance $(\mathrm{A})$. $\mathrm{O}$ grau de dependência espacial (GDE) foi calculado por meio da relação $\mathrm{C}_{0} /\left(\mathrm{C}_{0}+\mathrm{C}_{1}\right) * 100$, classificando-o como forte, para GDE $\leq 25 \%$; moderado, para GDE entre 25 e $75 \%$, e fraco, para GDE $>75 \%$ (CAMBARDELLA et al., 1994).

\section{RESULTADOS E DISCUSSÃO}

A análise estatística descritiva dos valores de $\mathrm{P}$ (Tabela 1) possibilitou verificar que, em geral, os teores médios encontram-se adequados, entretanto, a maioria das áreas ainda apresenta locais com $\mathrm{P}$ abaixo do teor crítico ( $<9 \mathrm{mg} \mathrm{dm}^{-3}$ para a classe 2 de argila), sendo considerados limitantes ao desenvolvimento das culturas pela CQFS-RS/SC (2004).

Os valores de $\mathrm{P}$ apresentaram grande amplitude, com teores máximos de 2,32 a 18,57 vezes maiores que os valores mínimos, indicando elevada potencialidade do manejo em sítio-específico preconizado na AP (AMADO et al., 2009). A variação dos teores é comprovada pelos altos valores de CV (>35\% em 63,34\% das áreas) e pela distribuição não normal dos dados, em 73,34\% das áreas. Altas variações nos teores de $\mathrm{P}$ concordam 
Tabela 1 - Estatística descritiva dos teores de fósforo $\left(\mathrm{P}, \mathrm{mg} \mathrm{dm}^{-3}\right)$ em áreas de Latossolos Vermelhos, amostradas a partir de diferentes dimensões de malhas amostrais.

\begin{tabular}{|c|c|c|c|c|c|c|c|c|c|c|c|}
\hline \multirow[t]{2}{*}{ Área } & \multirow{2}{*}{$\mathrm{n}$} & \multicolumn{4}{|c|}{--------------------------'Valores-------------------------- } & \multirow{2}{*}{ DP } & \multicolumn{4}{|c|}{ - } & \multirow{2}{*}{ Teste $\mathrm{W}^{(2)}$} \\
\hline & & Mínimo & Média & Máximo & $\mathrm{R}: \mathrm{M} / \mathrm{M}$ & & $\mathrm{CV}$ & $\mathrm{CP}$ & $\mathrm{Cs}$ & $\mathrm{Ck}$ & \\
\hline & & & & ------- & Amostral: & $\mathrm{x} 100 \mathrm{~m}$ & & & ------ & ----- & ------- \\
\hline 1 & 89 & 6,80 & 27,08 & 63,00 & 9,26 & 11,56 & 42,68 & 4,52 & 1,11 & 0,93 & $0,91 *$ \\
\hline 2 & 28 & 9,40 & 16,16 & 31,40 & 3,34 & 5,51 & 35,09 & 6,44 & 1,74 & 2,31 & $0,77^{*}$ \\
\hline 3 & 33 & 4,10 & 12,00 & 23,60 & 5,76 & 4,76 & 39,68 & 6,91 & 0,74 & 0,53 & $0,94 \mathrm{~ns}$ \\
\hline 4 & 40 & 10,90 & 21,06 & 40,30 & 3,70 & 7,65 & 36,30 & 5,74 & 0,81 & 0,19 & $0,93 *$ \\
\hline 5 & 49 & 10,40 & 19,47 & 32,50 & 3,13 & 4,61 & 23,69 & 3,38 & 0,44 & 0,42 & $0,98 \mathrm{~ns}$ \\
\hline 6 & 57 & 5,00 & 17,89 & 50,00 & 10,00 & 8,88 & 49,63 & 6,57 & 1,37 & 2,50 & $0,90^{*}$ \\
\hline 7 & 65 & 10,80 & 24,02 & 43,40 & 4,02 & 7,07 & 29,44 & 3,65 & 0,70 & 0,44 & $0,96 \mathrm{~ns}$ \\
\hline 8 & 47 & 8,70 & 16,74 & 32,60 & 3,75 & 5,32 & 31,77 & 4,63 & 1,03 & 0,87 & $0,92 *$ \\
\hline 9 & 46 & 5,40 & 15,80 & 40,90 & 7,57 & 8,10 & 51,28 & 7,56 & 1,40 & 2,29 & $0,88^{*}$ \\
\hline 10 & 41 & 3,90 & 8,19 & 16,70 & 4,28 & 2,51 & 30,68 & 4,79 & 1,05 & 2,45 & $0,94 *$ \\
\hline 1 & 45 & 13,00 & 19,82 & \begin{tabular}{l}
33,00 \\
\hdashline$----M$ \\
33
\end{tabular} & $\begin{array}{l}\text { Amostral: } \\
2,54\end{array}$ & $\begin{array}{l}x 142 \mathrm{~m} \\
4,25\end{array}$ & 21,47 & 3,20 & 1,22 & 1,60 & $0,90 *$ \\
\hline 2 & 75 & 6,80 & 13,88 & 41,00 & 6,03 & 6,61 & 47,59 & 5,50 & 1,83 & 4,36 & $0,83^{*}$ \\
\hline 3 & 45 & 13,00 & 20,93 & 35,00 & 2,69 & 4,81 & 22,97 & 3,42 & 1,25 & 1,54 & $0,89^{*}$ \\
\hline 4 & 43 & 13,00 & 23,19 & 41,00 & 3,15 & 6,36 & 27,44 & 4,18 & 0,76 & 0,25 & $0,95 \mathrm{~ns}$ \\
\hline 5 & 59 & 13,00 & 24,27 & 53,00 & 4,08 & 7,31 & 30,12 & 3,92 & 1,39 & 3,55 & $0,91 *$ \\
\hline 6 & 73 & 13,00 & 24,01 & 43,00 & 3,31 & 6,46 & 26,90 & 3,15 & 0,81 & 0,63 & $0,95^{*}$ \\
\hline 7 & 60 & 11,00 & 19,30 & 38,00 & 3,45 & 6,89 & 35,72 & 4,61 & 0,85 & 0,09 & $0,92^{*}$ \\
\hline 8 & 62 & 2,10 & 17,48 & 39,00 & 18,57 & 7,35 & 42,06 & 5,34 & 0,38 & 0,23 & $0,98 \mathrm{~ns}$ \\
\hline 9 & 83 & 5,60 & 25,82 & 53,00 & 9,46 & 10,79 & 41,79 & 4,59 & 0,07 & $-0,65$ & $0,98 \mathrm{~ns}$ \\
\hline 10 & 91 & 6,30 & 21,24 & 59,00 & 9,37 & 9,24 & 43,51 & 4,56 & 1,12 & 2,63 & $0,93^{*}$ \\
\hline 1 & 34 & 3,60 & 9,81 & -------M & $\begin{array}{l}\text { Amostral: } \\
7,31\end{array}$ & $\begin{array}{l}\text { x } 173 \mathrm{n} \\
4,98\end{array}$ & 50,82 & 8,72 & 1,35 & 2,41 & $0,88^{*}$ \\
\hline 2 & 36 & 2,70 & 4,80 & 10,00 & 3,70 & 1,56 & 32,57 & 5,43 & 1,35 & 2,20 & $0,88^{*}$ \\
\hline 3 & 51 & 3,60 & 12,87 & 32,10 & 8,92 & 6,76 & 52,57 & 7,36 & 1,06 & 0,95 & $0,92 *$ \\
\hline 4 & 33 & 3,50 & 11,41 & 24,80 & 7,09 & 4,40 & 38,57 & 6,71 & 1,13 & 1,66 & $0,92 *$ \\
\hline 5 & 27 & 4,10 & 9,91 & 19,10 & 4,66 & 3,68 & 37,10 & 7,14 & 0,74 & $-0,09$ & $0,93 \mathrm{~ns}$ \\
\hline 6 & 20 & 9,50 & 16,27 & 22,00 & 2,32 & 3,44 & 21,13 & 4,72 & $-0,04$ & $-0,80$ & $0,96 \mathrm{~ns}$ \\
\hline 7 & 24 & 5,00 & 11,88 & 30,70 & 6,14 & 6,96 & 58,57 & 11,96 & 1,57 & 2,13 & $0,82 *$ \\
\hline 8 & 31 & 2,90 & 8,25 & 18,40 & 6,34 & 4,01 & 48,61 & 8,73 & 0,85 & 0,21 & $0,93 \mathrm{~ns}$ \\
\hline 9 & 40 & 10,00 & 26,77 & 63,10 & 6,31 & 12,31 & 45,98 & 7,27 & 1,33 & 1,98 & $0,89 *$ \\
\hline 10 & 34 & 1,50 & 9,88 & 21,60 & 14,40 & 5,11 & 51,69 & 8,86 & 0,69 & 0,00 & $0,93^{*}$ \\
\hline
\end{tabular}

${ }^{(1)} \mathrm{n}$ : número de observações (pontos amostrais); R:M/M: relação entre valores máximos e mínimos; DP: desvio-padrão; CV(\%): coeficiente de variação; CP (\%): coeficiente de precisão; Cs: coeficiente de assimetria; Ck: coeficiente de curtose; ${ }^{(2)}$ Teste W: Teste de Shapiro-Wilk para distribuição normal, em que: $(*)$ significativo em níveis de $\mathrm{P}<0,05$ e (ns) não significativo. Quando for significativo, indica que a hipótese para distribuição normal é rejeitada.

com diversos estudos em Latossolos reportados na literatura (MONTANARI et al., 2008; AMADO et al., 2009; CHERUBIN et al., 2011; NANNI et al., 2011; BOTTEGA et al., 2013). De acordo com muitos dos autores acima citados, a elevada variação dos teores de $\mathrm{P}$ decorre das sucessivas aplicações de fertilizantes a taxa fixa na linha de semeadura, contribuindo para a manutenção e, ou, aumento da micro e macrovariabilidade espacial nas áreas.
Em relação ao $\mathrm{K}$ (Tabela 2 ), verificou-se que as áreas estudadas apresentaram teores acima do nível crítico $\left(60 \mathrm{mg} \mathrm{dm}^{-3}\right)$, classificados como altos (61 a $120 \mathrm{mg} \mathrm{dm}^{-3}$ ) e muito altos $\left(>120 \mathrm{mg} \mathrm{dm}^{-3}\right.$ ) (CQFS$\mathrm{RS} / \mathrm{SC}$, 2004). Altos teores de $\mathrm{K}$ também foram verificados por AMADO et al. (2009) e CHERUBIN et al. (2011) em Latossolos no sul do Brasil, e estão atrelados ao intenso intemperismo dos minerais primários (micas, feldspatos e feldspatoides) e 
Tabela 2 - Estatística descritiva dos teores de potássio $\left(\mathrm{K}, \mathrm{mg} \mathrm{dm}^{-3}\right)$ em áreas de Latossolos Vermelhos, amostradas a partir de diferentes dimensões de malhas amostrais.

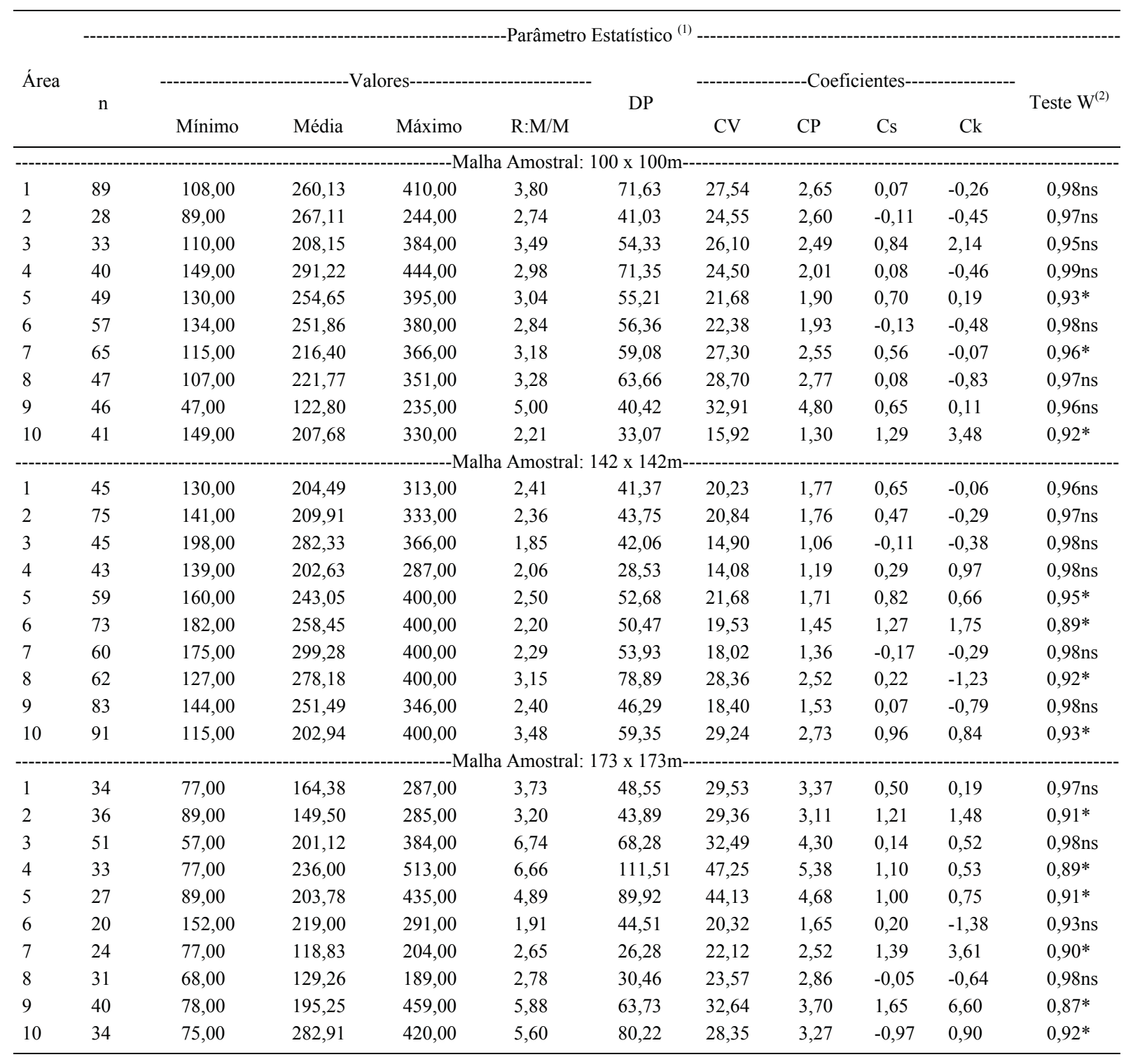

${ }^{(1)}$ n: número de observações (pontos amostrais); R:M/M: relação entre valores máximos e mínimos; DP: desvio-padrão; CV(\%): coeficiente de variação; CP (\%): coeficiente de precisão; Cs: coeficiente de assimetria; Ck: coeficiente de curtose; ${ }^{(2)}$ Teste W: Teste de Shapiro-Wilk para distribuição normal, em que: $\left(^{*}\right)$ significativo em níveis de $\mathrm{P}<0,05$ e (ns) não significativo. Quando for significativo, indica que a hipótese para distribuição normal é rejeitada.

secundários (illita, vermiculita e argilominerais interestratificados) que contém este elemento (ERNANI et al., 2007), associado ao histórico de sucessivas adubações, utilizando formulações com elevadas quantidades de $\mathrm{K}$ adicionadas às culturas.

Os teores máximos de $\mathrm{K}$ foram de 1,85 a 6,74 vezes maiores que os valores mínimos. Essa menor variação, em relação à observada no $\mathrm{P}$, é refletida pelos valores de CV, entre 15-35\% em
93,33\% das áreas, e pela distribuição de frequências normais, observadas em $56,67 \%$ das áreas estudadas. As menores variações do $K$, também verificadas por AMADO et al. (2009), CHERUBIN et al. (2011) e BOTTEGA et al. (2013), podem ser atribuídas à maior mobilidade do K no solo (ERNANI et al., 2007) em relação ao $\mathrm{P}$, minimizando a microvariabilidade a partir da concentração de fertilizantes potássicos na linha. Além disso, a rápida liberação do $\mathrm{K}$ presente 
nos restos culturais, dispostos uniformemente na área, contribui na redução da variabilidade desse atributo no solo.

Efetuada por meio dos semivariogramas, a análise geoestatística permitiu detectar que as áreas estudadas apresentaram diferentes escalas de variabilidade espacial para o $\mathrm{P}$ (Tabela 3 ) e para o K (Tabela 4), mesmo quando amostradas com a mesma dimensão de malha. Essa constatação é comprovada pelas distâncias em que os atributos apresentaram continuidade espacial (alcances), que variaram de 273 a $3.110 \mathrm{~m}$ para o $\mathrm{P}$ e de 315 a $3.280 \mathrm{~m}$ para o K, com grau de dependência espacial classificado de moderado a forte.

Em todas as malhas estudadas, verificaramse áreas em que os valores de $\mathrm{P}$ e K não apresentaram dependência espacial, caracterizando distribuições aleatórias (efeito pepita puro - EPP). O EPP pode ocorrer devido a erros de medidas, de amostragem ou microvariações não detectadas, considerando que o espaçamento de amostragem utilizado é maior que o necessário para detectar dependência espacial (CAMBARDELLA et al., 1994; WEBSTER \& OLIVER, 2007). Nesse sentido, observou-se que

Tabela 3 - Análise geoestatística dos teores de fósforo $\left(\mathrm{P}, \mathrm{mg} \mathrm{dm}^{-3}\right)$ em áreas de Latossolos Vermelhos, amostradas a partir de diferentes dimensões de malhas amostrais.

\begin{tabular}{|c|c|c|c|c|c|c|c|c|c|c|}
\hline \multirow{2}{*}{ Área } & \multirow{2}{*}{$\mathrm{n}^{(1)}$} & \multirow{2}{*}{ Efeito Pepita } & \multirow{2}{*}{ Patamar } & \multirow{2}{*}{ Alcance (m) } & \multirow{2}{*}{ Modelo } & \multirow{2}{*}{$\mathrm{SQR}^{(2)}$} & \multirow{2}{*}{$r^{2}$} & \multicolumn{2}{|c|}{-----GDE ${ }^{(3)}$} & \multirow{2}{*}{$\mathrm{CR}^{(4)}$} \\
\hline & & & & & & & & $\%$ & Classe & \\
\hline \multicolumn{11}{|c|}{---------------------------------------------------------------Malha Amostral: 100 x 100 m------------------------------------------------------------ } \\
\hline 1 & 89 & 18,00 & 286,00 & $1.711,00$ & Esférico & $1.024,00$ & 0,98 & 6,29 & Forte & 1,19 \\
\hline 2 & 28 & $\mathrm{EPP}^{(5)}$ & EPP & EPP & - & - & - & - & - & - \\
\hline 3 & 33 & 0,01 & 23,33 & 273,00 & Exponencial & 13,20 & 0,70 & 0,04 & Forte & 0,54 \\
\hline 4 & 40 & 30,20 & 67,57 & 431,00 & Esférico & 623,00 & 0,58 & 44,69 & Moderado & 0,88 \\
\hline 5 & 49 & EPP & EPP & EPP & - & - & - & - & - & - \\
\hline 6 & 57 & 23,81 & 47,63 & $2.643,00$ & Exponencial & 276,00 & 0,30 & 49,99 & Moderado & 0,41 \\
\hline 7 & 65 & 35,20 & 70,41 & $1.710,00$ & Esférico & 302,00 & 0,41 & 49,99 & Moderado & 1,16 \\
\hline 8 & 47 & 22,59 & 45,19 & $1.744,00$ & Esférico & 760,00 & 0,70 & 49,99 & Moderado & 0,98 \\
\hline 9 & 46 & 45,30 & 118,89 & $2.110,00$ & Esférico & 741,00 & 0,44 & 38,10 & Moderado & 0,63 \\
\hline 10 & 41 & EPP & EPP & $\mathrm{EPP}$ & - & - & - & - & - & - \\
\hline 1 & 45 & EPP & EPP & EPP & Ina Amostral: 1 & $\begin{array}{l}\mathrm{x} 142 \mathrm{~m}--- \\
-\end{array}$ & - & - & - & - \\
\hline 2 & 75 & 25,96 & 55,20 & 888,54 & Gaussiano & 458,00 & 0,94 & 47,03 & Moderado & 1,22 \\
\hline 3 & 45 & 1,60 & 54,20 & $1.913,00$ & Esférico & 498,00 & 0,94 & 2,95 & Forte & 2,06 \\
\hline 4 & 43 & 19,10 & 88,46 & $2.110,00$ & Esférico & 51,90 & 0,90 & 21,59 & Forte & 1,21 \\
\hline 5 & 59 & 40,00 & 80,01 & $2.110,00$ & Esférico & $1.633,00$ & 0,34 & 49,99 & Moderado & 0,62 \\
\hline 6 & 73 & EPP & EPP & EPP & - & - & - & - & - & - \\
\hline 7 & 60 & 35,10 & 101,11 & $2.099,00$ & Gaussiano & 453,00 & 0,88 & 34,71 & Moderado & 0,76 \\
\hline 8 & 62 & EPP & EPP & EPP & - & - & - & - & - & - \\
\hline 9 & 83 & 28,90 & 136,50 & 850,00 & Esférico & $1.051,00$ & 0,95 & 21,17 & Forte & 1,48 \\
\hline 10 & 91 & 47,00 & 163,30 & $3.110,00$ & Esférico & 931,00 & 0,73 & 28,78 & Moderado & 1,34 \\
\hline & & & & ------------M & lha Amostral: & $3 \times 173 m--$ & ----- & ------ & - & ------ \\
\hline 1 & 34 & 6,20 & 60,45 & $2.622,00$ & Esférico & 345,00 & 0,66 & 10,26 & Forte & 0,90 \\
\hline 2 & 36 & EPP & EPP & EPP & - & - & - & - & - & - \\
\hline 3 & 51 & EPP & EPP & EPP & - & - & - & - & - & - \\
\hline 4 & 33 & 0,01 & 21,42 & 642,00 & Exponencial & 279,00 & 0,52 & 0,05 & Forte & 0,91 \\
\hline 5 & 27 & EPP & EPP & EPP & - & - & - & - & - & - \\
\hline 6 & 20 & 9,32 & 18,65 & $2.110,00$ & Esférico & 428,00 & 0,38 & 49,97 & Moderado & 0,87 \\
\hline 7 & 24 & 0,10 & 55,60 & 399,00 & Esférico & 108,00 & 0,74 & 0,18 & Forte & 1,02 \\
\hline 8 & 31 & 0,01 & 17,13 & 430,00 & Esférico & 38,00 & 0,80 & 0,06 & Forte & 0,96 \\
\hline 9 & 40 & EPP & EPP & EPP & - & - & - & - & - & - \\
\hline 10 & 34 & 0,01 & 29,86 & 605,00 & Esférico & 45,00 & 0,69 & 0,03 & Forte & 0,85 \\
\hline
\end{tabular}

${ }^{(1)} \mathrm{n}$ : número de observações (pontos amostrais); ${ }^{(2)} \mathrm{SQR}$ : soma do quadrado dos resíduos; ${ }^{(3)} \mathrm{GDE}$ : grau de dependência espacial; ${ }^{(4)} \mathrm{CR}$ : coeficiente de regressão (validação cruzada) $;{ }^{(5)}$ EPP: efeito pepita puro. 
Tabela 4 - Análise geoestatística dos teores de potássio $\left(\mathrm{K}, \mathrm{mg} \mathrm{dm}^{-3}\right)$ em áreas de Latossolos Vermelhos, amostradas a partir de diferentes dimensões de malhas amostrais.

\begin{tabular}{|c|c|c|c|c|c|c|c|c|c|c|}
\hline \multirow{2}{*}{ Área } & \multirow{2}{*}{$\mathrm{n}^{(1)}$} & \multirow{2}{*}{ Efeito Pepita } & \multirow{2}{*}{ Patamar } & \multirow{2}{*}{ Alcance (m) } & \multirow{2}{*}{ Modelo } & \multirow{2}{*}{$\mathrm{SQR}^{(2)}$} & \multirow{2}{*}{$r^{2}$} & \multicolumn{2}{|c|}{----'GDE ${ }^{(3)}$} & \multirow{2}{*}{$\mathrm{CR}^{(4)}$} \\
\hline & & & & & & & & $\%$ & Classe & \\
\hline 1 & 89 & $2.500,00$ & $6.011,00$ & 924,00 & $\begin{array}{l}\text { Amostral: } 100 \\
\text { Exponencial }\end{array}$ & $6,12 \times 10^{6}$ & 0,55 & 41,59 & Moderado & 0,81 \\
\hline 2 & 28 & $\operatorname{EPP}^{(5)}$ & EPP & EPP & - & - & - & - & - & - \\
\hline 3 & 33 & 910,00 & $4.930,00$ & 866,00 & Esférico & $9,35 \times 10^{4}$ & 0,96 & 18,46 & Forte & 1,29 \\
\hline 4 & 40 & $2.640,00$ & $5.823,00$ & 623,00 & Esférico & $3,91 \times 10^{6}$ & 0,98 & 45,34 & Moderado & 1,247 \\
\hline 5 & 49 & $1.630,00$ & $6.370,00$ & 1699,00 & Esférico & $4,74 \times 10^{5}$ & 0,97 & 25,59 & Moderado & 0,926 \\
\hline 6 & 57 & EPP & EPP & EPP & - & - & - & - & - & - \\
\hline 7 & 65 & 960,00 & $4.421,00$ & 649,00 & Esférico & $3,44 \times 10^{6}$ & 0,94 & 21,71 & Forte & 1,21 \\
\hline 8 & 47 & 950,00 & $7.010,00$ & 985,00 & Esférico & $2,38 \times 10^{6}$ & 0,96 & 13,55 & Forte & 1,20 \\
\hline 9 & 46 & $1.212,00$ & $2.425,00$ & 3030,00 & Exponencial & $1,51 \times 10^{5}$ & 0,58 & 49,98 & Moderado & 0,33 \\
\hline 10 & 41 & EPP & ЕРP & EPP & - & - & - & - & - & - \\
\hline & & & & ----------Mal & Amostral: 142 & 142m--------- & ------- & -------- & ---------------- & ------ \\
\hline 1 & 45 & 578,00 & $1.809,00$ & 553,00 & Esférico & $2,66 \times 10^{4}$ & 0,94 & 31,95 & Moderado & 1,32 \\
\hline 2 & 75 & 22,00 & $1.998,00$ & 344,00 & Esférico & $9,16 \times 10^{5}$ & 0,75 & 1,10 & Forte & 0,73 \\
\hline 3 & 45 & 987,00 & $3.741,00$ & 2684,00 & Esférico & $3,86 \times 10^{5}$ & 0,81 & 26,38 & Moderado & 0,95 \\
\hline 4 & 43 & 1,00 & 887,50 & 315,23 & Esférico & $4,76 \times 10^{4}$ & 0,81 & 0,11 & Forte & 0,91 \\
\hline 5 & 59 & 593,00 & $3.040,00$ & 489,00 & Exponencial & $3,47 \times 10^{5}$ & 0,80 & 19,51 & Forte & 1,04 \\
\hline 6 & 73 & EPP & EPP & EPP & - & - & - & - & - & - \\
\hline 7 & 60 & $1.660,00$ & $6.041,00$ & 2589,00 & Esférico & $2,20 \times 10^{6}$ & 0,88 & 27,48 & Moderado & 0,86 \\
\hline 8 & 62 & $4.780,00$ & $30.660,00$ & 4091,00 & Gaussiano & $2,08 \times 10^{6}$ & 0,93 & 15,59 & Forte & 0,81 \\
\hline 9 & 83 & $1.775,00$ & $3.551,00$ & 3110,00 & Esférico & $3,14 \times 10^{6}$ & 0,42 & 49,99 & Moderado & 0,49 \\
\hline 10 & 91 & $2.630,00$ & $5.261,00$ & 3110,00 & Esférico & $1,60 \times 10^{6}$ & 0,34 & 49,99 & Moderado & 0,30 \\
\hline 1 & 34 & EPP & EPP & EPP & - & - & - & - & - & - \\
\hline 2 & 36 & EPP & EPP & EPP & - & - & - & - & - & - \\
\hline 3 & 51 & $3.480,00$ & $11.070,00$ & 3280,00 & Gaussiano & $2,96 \times 10^{6}$ & 0,78 & 31,44 & Moderado & 0,32 \\
\hline 4 & 33 & 10,00 & $12.990,00$ & 395,00 & Esférico & & 0,76 & 0,08 & Forte & \\
\hline 5 & 27 & $4.520,00$ & $30.140,00$ & 2771,00 & Gaussiano & $4,89 \times 10^{7}$ & 0,80 & 15,00 & Forte & 1,41 \\
\hline 6 & 20 & 340,00 & $2.423,00$ & 508,00 & Esférico & $3,25 \times 10^{4}$ & 0,97 & 14,03 & Forte & 0,94 \\
\hline 7 & 24 & 1,00 & 745,80 & 573,00 & Esférico & $3,76 \times 10^{4}$ & 0,87 & 0,13 & Forte & 0,89 \\
\hline 8 & 31 & 1,00 & $1.158,00$ & 865,00 & Esférico & & 0,85 & 0,09 & Forte & \\
\hline 9 & 40 & EPP & EPP & EPP & - & - & - & - & - & - \\
\hline 10 & 34 & EPP & ЕРP & EPP & - & - & - & - & - & - \\
\hline
\end{tabular}

${ }^{(1)} \mathrm{n}$ : número de observações (pontos amostrais); ${ }^{(2)} \mathrm{SQR}$ : soma do quadrado dos resíduos; ${ }^{(3)} \mathrm{GDE}$ : grau de dependência espacial; ${ }^{(4)} \mathrm{CR}$ : coeficiente de regressão (validação cruzada) ${ }^{(5)} \mathrm{EPP}$ : efeito pepita puro.

há uma tendência de aumento na ocorrência de distribuições aleatórias, à medida que aumenta a dimensão da malha amostral, confirmando que as malhas amostrais utilizadas possuem pontos muito espaçados e/ou em número insuficientes para detectar a dependência que, se existir, será manifestada a distâncias menores que o menor espaçamento utilizado entre amostras.

A não detecção da dependência espacial, ou o comportamento aleatório do P e K, observado em $33,33 \%$, das áreas estudadas, deve-se ao fato de que esses atributos são altamente afetados por práticas de manejo (adubações em linha, exportações via colheita, sistema plantio direto e cultura cultivada), condicionando a variações mais pronunciadas em curtas distâncias (NANNI et al., 2011). Resultados semelhantes foram obtidos por CORÁ \& BERALDO (2006) e NANNI et al. (2011), demonstrando que a coleta de uma amostra por ha não foi suficiente para captar a variabilidade espacial de P e K, indicando a necessidade do uso de malhas mais adensadas para recomendar fertilização com maior acurácia. Os resultados obtidos neste estudo também concordam com a realidade verificada nas áreas comerciais 
que adotam a AP nos Estados Unidos, em que as amostragens de solo, comumente utilizando malhas de 100x100m (uma amostra por ha), não são eficientes na detecção das diferentes escalas de variação dos atributos do solo, em especial de P e K (KERRY et al., 2010).

A partir dos resultados, poder-se-ia inferir que as malhas amostrais utilizadas possibilitaram captar a variabilidade espacial do $\mathrm{P}$ e K para a maioria das áreas estudadas, entretanto, tal afirmação merece cautela, uma vez que os semivariogramas foram gerados utilizando um limitado número de pontos (n), o que pode comprometer a consistência dos resultados. De acordo com LANDIM (2006), para utilizar a geoestatística, indica-se que sejam adotados no mínimo 30 pares para cada distância estudada.

Portanto, de acordo com os resultados e argumentações apresentadas, afirma-se que as dimensões de malhas amostrais, comumente utilizadas nas áreas de Latossolos manejados com AP no sul do Brasil, de modo geral, possibilitaram captar as diferentes escalas da variabilidade espacial de $\mathrm{P}$ e K. Apesar disso, recomenda-se evitar a utilização de malhas amostrais que condicionem a obtenção de poucas amostras nas áreas, uma vez que limita a acurácia da caracterização e o manejo da variabilidade espacial dos atributos analisados.

Ressalta-se também que a caracterização e manejo da microvariabilidade espacial dos teores de $\mathrm{P}$ e K, observada em parte das áreas e condicionada pelas práticas de manejo adotadas, exigem a utilização de malhas amostrais menores (mais de uma amostra por ha) do que as utilizadas atualmente, associando, assim, menores distâncias entre amostras e maior número de amostras coletadas na área. Dessa forma, não é possível extrapolar regionalmente um modelo confiável de variabilidade espacial destes atributos. Entretanto, o estudo fornece uma noção geral da escala de variação de $\mathrm{P}$ e $\mathrm{K}$, constituindo-se um importante referencial que poderá ser considerado no planejamento de futuras estratégias de amostragem de solo a serem adotadas nas áreas de AP da região estudada.

Os resultados demonstraram que a acurácia dos mapeamentos da variabilidade espacial de $\mathrm{P}$ e $\mathrm{K}$ no solo e, consequentemente, o sucesso de intervenções localizadas em sítios-específicos depende da utilização de malhas amostrais adensadas, que possibilitem coletar um número de amostras suficiente para captar as diferentes escalas de variabilidade que ocorrem na área. Dessa forma, verifica-se que um dos grandes desafios da AP está na busca por metodologias alternativas, que permitam obter mapas acurados de atributos do solo, gerados a partir de maior número de informações possíveis, em menor tempo e com baixo custo.

Nesse sentido, o uso de sensores baseados em técnicas, como a espectroscopia do infravermelho próximo (NIR) e visível (VIS), tem apresentado resultados promissores na estimativa de atributos do solo, como o P e o K (KODAIRA \& SHIBUSAWA, 2013) e tem sido apontado com um dos principais direcionamentos da AP.

\section{CONCLUSÃO}

As dimensões de malhas amostrais utilizadas nas áreas manejados com AP, sob procedimentos geoestatísticos, são eficientes para captar as diferentes escalas da variabilidade espacial dos teores de P e K no solo. Entretanto, recomendase evitar a utilização de malhas amostrais que condicionem a obtenção de poucas amostras nas áreas e, consequentemente, a caracterização e manejo da variabilidade espacial dos atributos analisados com menor acurácia.

A variabilidade espacial expressa em curtas distâncias deverá ser considerada em futuros planos de amostragem nas áreas manejadas com AP, indicando a necessidade da utilização de malhas amostrais menores (mais uma amostra por ha) em relação às utilizadas atualmente.

\section{AGRADECIMENTOS}

Ao Programa de Pós-graduação em Agronomia: Agricultura em Ambiente da Universidade Federal de Santa Maria (UFSM). A Coordenação Aperfeiçoamento de Pessoal de Nível Superior (CAPES) pela concessão da bolsa ao primeiro autor.

\section{REFERÊNCIAS}

AMADO, T.J.C. et al. Atributos químicos e físicos de Latossolos e sua relação com os rendimentos de milho e feijão irrigados. Revista Brasileira de Ciência do Solo, v.33, n.4, p.831843, 2009. Disponível em: <http://www.scielo.br/pdf/rbcs/ v33n4/08.pdf>. Acesso em: 20 nov. 2012. doi:10.1590/S010006832009000400008

BOTTEGA, E.L. et al. Variabilidade espacial de atributos do solo em sistema de semeadura direta com rotação de culturas no cerrado brasileiro. Revista Ciência Agronômica, v.44, n.1, p.19, 2013. Disponível em: <http://www.scielo.br/pdf/rca/v44n1/ a01v44n1.pdf>.Acesso em: 2 jan. 2013.doi: 10.1590/S180666902013000100001

CAMBARDELLA, C.A. et al. Fieldscale variability of soil properties in central Iowa soils. Soil Science Society of America Journal, v.8, n.6, p.1501-1511, 1994. Disponível em: <http:// ddr.nal.usda.gov./bitstream/10113/18168/1/IND20518015.pdf>. Acesso em: 12 set. 2009. 
CHERUBIN, M.R. et al. Caracterização e estratégias de manejo da variabilidade espacial dos atributos químicos do solo utilizando a análise dos componentes principais. Enciclopédia Biosfera, v.7, p.196-210, 2011. Disponível em: <http://www.conhecer.org. br/enciclop/2011b/ ciencias $\% 20$ agrarias/caracterizacao $\% 20 \mathrm{e} \% 20$ estrategias.pdf $>$. Acesso em: 12 dez. 2011.

COMISSÃO DE QUÍMICA E FERTILIDADE DO SOLO (CQFS$\mathrm{RS} / \mathrm{SC}$ ). Manual de adubação e calagem para os estados do Rio Grande do Sul e Santa Catarina. Porto Alegre: Sociedade Brasileira de Ciência do Solo - Núcleo Regional Sul, 2004. 400p.

CORÁ, J.E.; BERALDO, J.M.G. Variabilidade espacial de atributos do solo antes e após calagem e fosfatagem em doses variadas na cultura de cana-de-açúcar. Engenharia Agrícola, v.26, n.2, p.374-387, 2006. Disponível em: <http:/www.scielo.br/ pdf/eagri/v26n2/05.pdf>. Acesso em: 10 set. 2012. doi:10.1590/ S0100-69162006000200005.

ERNANI, P.R. et al. Potássio. In: NOVAIS, R.F. et al. (Eds.). Fertilidade do solo. Viçosa-MG: Sociedade Brasileira de Ciência do Solo, 2007. p.551-594.

KERRY, R. et al. Sampling in precision agriculture. In: OLIVER, M.A. (Org.). Geostatistical applications for precision agriculture. Heidelberg: Springer-Verlag, 2010. p.35-63.

KODAIRA, M.; SHIBUSAWA, S. Using a mobile realtime soil visible-near infrared sensor for high resolution soil property mapping. Geoderma, v. 199, n.5, p.64-79, 2013. Disponível em: <http://www.sciencedirect.com/science/article/ pii/S0016706112003412>. Acesso em: 27 nov. 2012. doi: http:// dx.doi.org/10.1016/j.geoderma.2012.09.007.

LANDIM, P.M.B. Sobre geoestatística e mapas. Terræ Didatica, v.2, n.1, p.19-33, 2006. Disponível em: <http://www.ige.unicamp. br/terraedidatica/v2/pdfv2/t didatica_2006_v02n01_p019-033 landim.pdf $>$. Acesso em: $9 \overline{\mathrm{dez}}$. 2012.
MONTANARI, R. et al. Variabilidade espacial de atributos químicos em Latossolo e Argissolos. Ciência Rural, v.38, n.5, p.1266-1272, 2008. Disponível em: <http://www.scielo.br/pdf/ cr/v38n5/a10v38n5.pdf>. Acesso em: 11 nov. 2012. doi:10.1590/ S0103-84782008000500010.

MONTANARI, R. et al. The use of scaled semivariograms to plan soil sampling in sugarcane fields. Precision Agriculture, v.13, n.5, p.542-552, 2012. Disponível em: <http://link.springer.com/ content/pdf/10.1007\%2Fs11119-012-9265-6.pdf $>$. Acesso em: 10 out. 2012.doi:10.1007/s11119-012-9265-6.

NANNI, M.R. et al. Optimum size in grid soil sampling for variable rate application in site-specific management. ScientiaAgrícola, v.68, n.3, p.386-392, 2011. Disponível em: <http://www.scielo. br/pdf/sa/v68n3/v68n3a17.pdf>. Acesso em: 18 dez. 2012. doi: 10.1590/S0103-90162011000300017.

SANTI, A.L. et al. É chegada a hora da integração do conhecimento. Revista Plantio Direto, v.129, n.1, p.24-30, 2009. Disponível em: <http://www.plantiodireto.com.br/?body=cont_int\&id=907>. Acesso em: 18 nov. 2012.

SOUZA, Z.M. de. et al. Otimização amostral de atributos de latossolos considerando aspectos solo-relevo. Ciência Rural, v.36, n.3 p.829-836, 2006. Disponível em: <http://www.scielo.br/pdf/cr/ v36n3/a16v36n3.pdf>. Acesso em: 25 nov. 2011. doi: 10.1590/ S0103-84782006000300016.

STRECK, E.V. et al. Solos do Rio Grande do Sul. 2.ed. Porto Alegre: EMATER/RS-ASCAR, 2008. 222p.

WEBSTER, R.; LARK, M. Field sampling for environmental science and management. London: Routledge. 2012. 200p.

WEBSTER, R.; OLIVER, M.A. Geostatistics for environmental scientists. 2.ed. Chichester: John Wiley\& Sons, 2007. 330p. 